\title{
Assessment of "fresh" versus "macerated" as accurate markers of time since intrauterine fetal demise in low-income countries
}

\author{
Katherine J. Gold a,b,*, Abdul-Razak S. Abdul-Mumin ${ }^{\mathrm{c}}$, Martha E. Boggs a \\ Henry S. Opare-Addo ${ }^{c}$, Richard W. Lieberman ${ }^{\text {b,d }}$ \\ a Department of Family Medicine, University of Michigan, Ann Arbor, USA \\ b Department of Obstetrics and Gynecology, University of Michigan, Ann Arbor, USA \\ c Department of Obstetrics and Gynaecology, College of Health Sciences, Kwame Nkrumah University of Science and Technology, Komfo Anokye Teaching Hospital, Kumasi, Ghana \\ d Department of Pathology, University of Michigan, Ann Arbor, USA
}

\section{A R T I C L E I N F O}

\section{Article history:}

Received 23 August 2013

Received in revised form 3 December 2013

Accepted 25 February 2014

\section{Keywords:}

Fetal death

Fetal pathology

Ghana

Low-income country

Maceration

Stillbirth

Sub-Saharan Africa

\begin{abstract}
A B S T R A C T
Objective: To compare provider assessment of fetal maceration with death-to-delivery interval to evaluate the reliability of appearance as a proxy for time of death. Methods: Cohort chart abstraction was performed for all stillbirth deliveries at or above 28 weeks of gestation during a 1-year period in a teaching hospital in Ghana. Results: Of 470 stillborn infants, 337 had adequate data for analysis. Of 47 fetuses alive on admission with death-to-delivery intervals estimated to be less than 8 hours (expected to be reported as fresh), 14 (30\%) were actually reported as macerated. Of 94 cases in which the fetus was deceased on admission with death-to-delivery interval of more than 8 hours (expected to be macerated), 17 (18\%) were described as fresh. Conclusion: Provider description of fetal appearance may be an unreliable indicator for time since fetal death. The findings have significant implications for stillbirth prevention and assessment.
\end{abstract}

(c) 2014 International Federation of Gynecology and Obstetrics. Published by Elsevier Ireland Ltd. All rights reserved.

\section{Introduction}

Stillbirth remains a severely understudied and grossly underreported problem in low-income countries [1,2]. Efforts to reduce unacceptably high stillbirth rates in low-income nations typically distinguish between deaths occurring prepartum (before labor) and those occurring intrapartum (during labor) [3]. Advocates have long assumed that fresh stillbirths occur shortly before delivery and so might be preventable based on changes in care, resources, education, or medical access [4-7]. These have often been considered the first priority for researchers because they may be "near misses," which-in theory-might be avoidable losses if access to adequate and appropriate care were available to the mother in time.

A reliable method to identify the approximate time of death and the death-to-delivery interval is challenging to obtain in low-resource settings. In community studies, verbal autopsies utilize interviews with families to register out-of-hospital births and fetal deaths and record the last time that the mother perceived fetal movement as a

\footnotetext{
* Corresponding author at: Department of Obstetrics and Gynecology, University of Michigan, 1018 Fuller Street, Ann Arbor, MI 48104-1216, USA. Tel.: +1 734998 7120x323; fax: +1 7349987335 .

E-mail address: ktgold@umich.edu (K.J. Gold).
}

proxy for time of death $[8,9]$. In facility-based deliveries, death-todelivery interval is generally based on fetal appearance, as assessed by the physician, nurse, or midwife at delivery. A "macerated" fetus shows skin and soft-tissue changes (skin discoloration or darkening, redness, peeling, and breakdown) suggesting death was well before delivery (prepartum) [1,10]. A "fresh" fetus lacks such skin changes and is presumed to have died much more recently (intrapartum). Recent case series from low-income countries have described cohorts of stillbirths delivered in hospital settings and have relied on fetal appearance to classify the death as prepartum versus intrapartum [11-13]. Unfortunately, there has been no research in low-income countries to study whether this is, in fact, a valid method of assessment.

The aim of the present study was to evaluate third-trimester stillbirths at a teaching hospital in Ghana over 1 year to compare provider assessment of fetal status at delivery (fresh or macerated) with the time between death and delivery according to medical records.

\section{Materials and methods}

From June 8, 2011, to June 12, 2012, we identified all stillbirths delivered at Komfo Anokye Teaching Hospital (KATH), which is one of the largest teaching hospitals in Ghana and is located in the center of the second largest city, Kumasi. We used WHO criteria to define 
stillbirth: fetal deaths delivered at 28 or more gestational weeks without signs of life at delivery [14]. The study was part of a broader investigation of risk factors associated with stillbirth. We hypothesized that the labeling of a fetus as fresh would correlate well with less than 8 hours since death, and macerated would correlate with at least 8 hours between death and delivery. The study was approved by the institutional review board at the University of Michigan and the Committee on Human Research Publication and Ethics of the Kwame Nkrumah University of Science and Technology in Kumasi, which governs research at KATH. Written informed consent was not required and data were de-identified. Reporting was based on STROBE guidelines [17].

The primary reviewer (A-R.S.A-M., a physician)visited the wards several times weekly throughout the year to collect data from paper charts and to discuss cases with the midwives on duty in order to fillin missing data and assess issues with staffing and personnel availability during the time of the patient's stay. For each fetal death, the reviewer abstracted the mother's chart to identify maternal demographics and past medical history; prior pregnancy outcomes; prenatal care and labs in the current pregnancy; referral source; admission data; delivery information; complications; and potential contributors to death. A second reviewer (K.J.G., a physician or M.E.B., the research coordinator) re-abstracted data from approximately $30 \%$ of charts to ensure accuracy, to fill-in missing data points, and to confirm outlying data. Data were initially recorded on a written datasheet and then entered into a computer database (Access version 14; Microsoft, Redmond, WA, USA) and triple-checked for data entry accuracy.

In most cases, we could identify whether the fetus was alive at admission to the hospital (yes/no) and the status on admission (whether in labor and whether membranes intact). Time of last fetal movement and time of labor onset were not documented for most cases, so we could rarely identify whether pre-hospital deaths were prepartum or intrapartum. We also coded whether the fetus was identified as fresh or macerated based on the delivering team's (physician or nurse-midwife) assessment of the infant recorded in the paper chart.

For all deaths, we collected information (when available) documenting day and time of admission, last documented positive fetal heart rate (FHR-recorded at the study hospital via ultrasound, Doppler, or fetoscope), time when intrauterine fetal demise (IUFD) was diagnosed, and time of delivery. While we had admission and delivery dates for most infants, data on last FHR and time of fetal death were limited, and in most cases applied to infants who were alive on admission.

In some cases, it was unclear from charts whether the fetus was alive on admission, and not all deliveries listed fetal condition at delivery; therefore, from the initial 470 fetal deaths, we restricted our analysis to cases for which these data were available. This left 337 stillborn fetuses in the cohort for analysis (72\% of the original dataset).

When the fetus was dead on admission, we assigned the time of death as the time of admission unless we had additional information (e.g. ultrasound prior to admission) that confirmed the time of IUFD. If we had an ultrasound documenting a live infant prior to admission, we calculated both a minimum death-to-delivery interval (time from IUFD diagnosis until delivery) and a maximum death-to-delivery interval ( 1 hour after the last positive FHR was recorded).

When the fetus was alive on admission and the FHR was not rechecked before delivery, we assigned the time of death as 1 hour after the last documented FHR. If the infant was alive on admission and then diagnosed as IUFD during labor, we calculated a minimum death-todelivery interval as time between IUFD confirmation and delivery. We calculated the maximum death-to-delivery interval as 1 hour after the last positive FHR and delivery. Sample cases illustrating calculation of minimum and maximum death-to-delivery intervals are described in Fig. 1.

We used 8 hours as our cutoff time based on the most commonly used maceration criteria described by Langley [15]; this is also the timing mentioned in the practice guidelines for perinatal autopsy for the Autopsy Committee of the College of American Pathologists [16]. If both the minimum and the maximum death-to-delivery intervals were less than 8 hours or both were more than 8 hours, the classification was straightforward. In a small number of cases, the minimum death-to-delivery interval was less than 8 hours and the maximum was more than 8 hours; in such cases, we reviewed the clinical scenario and determined the most probable timing.

Analysis to evaluate summary statistics was performed using STATA/ IC version 11.0 (StataCorp, College Station, TX, USA).

\section{Results}

There were 465 mothers who delivered a stillborn fetus at 28 weeks or later at KATH during the study period (Table 1). Two-thirds of pregnancies were dated by fetal ultrasound and the rest by last menstrual period. Of these mothers, 443 (95\%) had a singleton pregnancy, 20 had twins, and 2 had triplets. In most cases with multiples, only 1 fetus died. In 5 cases (all twin deliveries), both fetuses died. This resulted in a total of 470 stillborn fetuses. As noted, the analysis was restricted to the 337 fetuses with adequate data for analysis. The maternal demographics of this subgroup were not substantially different from those of the full group of mothers in the dataset.

For the 71 fetuses identified as being alive on admission, the time between death and delivery ranged from 0 to 275 hours (Fig. 2). In 47 cases, the death-to-delivery interval was less than 8 hours: 33 (70\%) fetuses were fresh and 14 (30\%) were macerated. In 20 cases, the death-to-delivery interval was more than 8 hours, half of which were fresh and half macerated. In 4 cases, the death-to-delivery interval (more than or less than 8 hours) could not be definitively determined, so these cases were not analyzed.

Of the 266 fetuses that were dead on admission, 172 had less than 8 hours from admission to delivery but we could not rule out the possibility that the fetus had died long before the mother presented to the hospital. Therefore, these cases were not analyzed. Of the 94 fetuses with a death-to-delivery interval of at least 8 hours, 17 (18\%) were reported as fresh, including many known to have been dead 24 hours or more, and 77 (82\%) were reported as macerated.

The Wigglesworth criteria for cause of death in cases of stillbirth exclude fetuses with visible congenital anomalies $[18,19]$. We chose not to exclude fetuses with anomalies $(n=21)$ because these were a small subset of the overall total and their classifications were not significantly different from the overall set of stillbirths.

\section{Discussion}

The present study demonstrated that appearance may not be an accurate proxy for death-to-delivery interval or prepartum versus intrapartum demise. Particularly among fetuses noted to be alive on admission to the hospital, one-third of those that would be anticipated to be fresh stillbirths were reported as macerated, and half of those that would be expected to be macerated were actually described as fresh.

When a fetus dies in utero, there are changes in the skin and tissuestermed fetal maceration. This process takes place entirely in the womb and stops once the fetus is delivered $[10,16]$. The phenomenon of maceration was first described in 1922 as loss of the vernix and then skin peeling [20]. In 1971, Langley [15] discussed levels of maceration and noted that peeling skin is associated with death of at least 8 hours; this is the most common classification system utilized. Practice guidelines for perinatal and pediatric autopsy by the Autopsy Committee of the College of American Pathologists also uses the 8-hour cutoff [16]. Genest and Singer [20] identified 60 fetuses for which exact times of death and delivery were known and photographs were available. The authors described detailed changes in skin color, desquamation, and collapse of the skull based on death-to-delivery interval. Early signs of maceration (desquamation of more than $1 \mathrm{~cm}$ and red/brown cord discoloration) began at 6 hours post-death. However, in their study (the only one in the literature to make this comparison), death- 
DECEASED ON ADMISSION
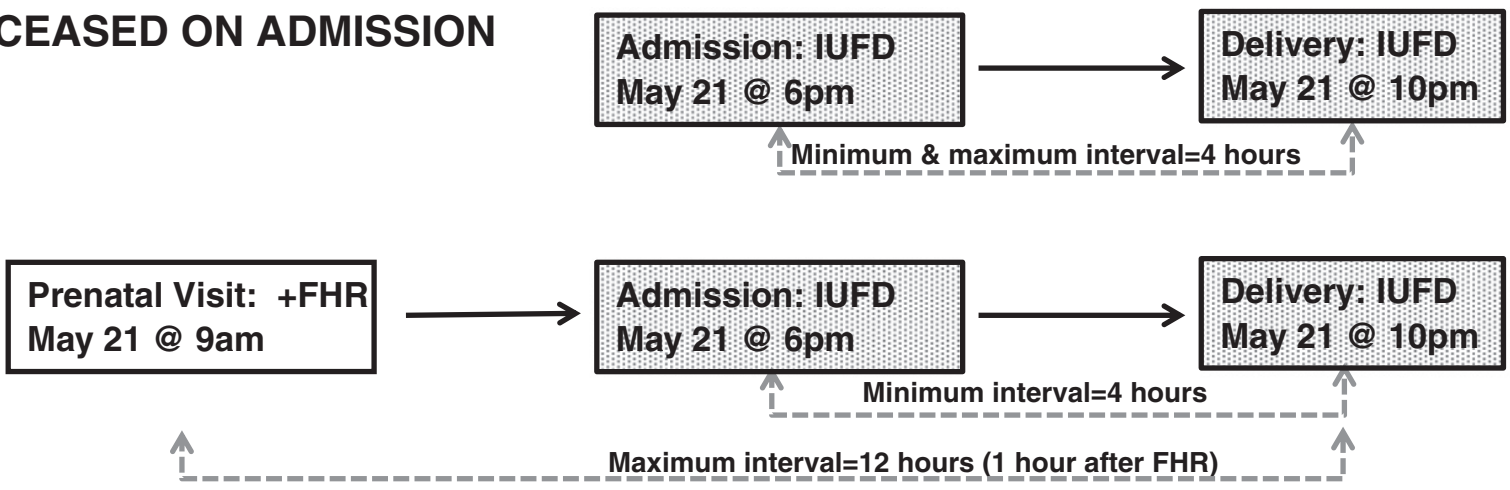

ALIVE ON ADMISSION
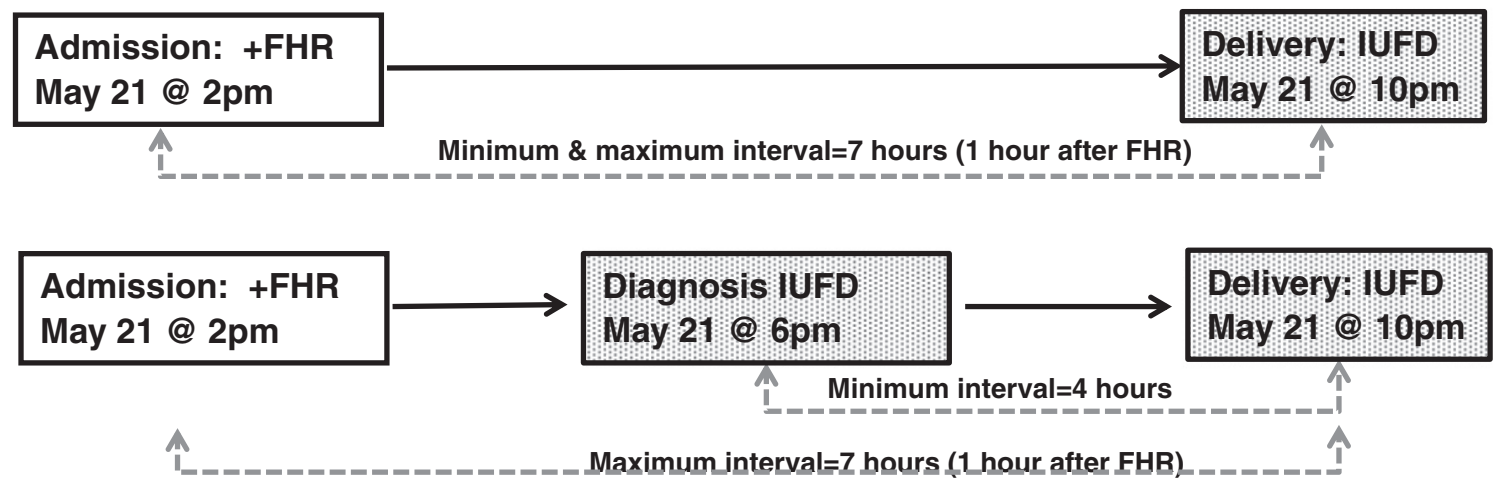

Fig. 1. Calculation of minimum and maximum death-to-delivery intervals for sample cases. Abbreviations: IUFD, intrauterine fetal demise; FHR, fetal heart rate.

to-delivery interval was miscalculated in almost one-third of 26 "test" photographs of stillborn fetuses. These same criteria were recently used in a major US study on stillbirth [21]. A key reference book for fetal pathology also mentions 6-8 hours as the earliest point when signs of maceration are seen [22].

Most studies of stillbirth that evaluate maceration do so without detailing a specific definition. Lawn et al. [1] reported that a fresh stillbirth implies death less than 12 hours before delivery, but this appears to be a clinical judgment. Maceration is a subjective diagnosis; while all providers are likely to describe a fetus with extensive skin desquamation

Table 1

Demographics of women $(n=465)$ who delivered stillborn fetuses. ${ }^{a}$

\begin{tabular}{ll}
\hline Characteristic & Value \\
\hline Age, y & $29 \pm 6(14-46)$ \\
Marital status & \\
$\quad$ Married & $194(41.7)$ \\
Living together & $92(19.8)$ \\
Never married & $95(20.4)$ \\
Widowed & $1(0.2)$ \\
Data missing & $83(17.8)$ \\
Primary residence & \\
Urban & $317(68.2)$ \\
Rural & $44(9.5)$ \\
Data missing & $104(22.4)$ \\
Education & \\
None & $65(14.0)$ \\
Primary school & $165(35.5)$ \\
Middle or junior secondary school & $161(34.6)$ \\
Senior secondary school or higher & $38(8.2)$ \\
Data missing & $36(7.7)$ \\
\hline
\end{tabular}

a Values are given as mean $\pm \mathrm{SD}$ (range) or number (percentage). and skull collapse as macerated, categorization may be less clear for a fetus with minor skin changes or skin slippage.

Maceration can also be an imprecise predictor of timing of death for technical reasons. High microbial load in the amniotic fluid, long duration of hypoxia prior to actual death, and maternal fever might all contribute to more maceration than would otherwise be expected for a certain death-to-delivery interval. Many studies describe the rate of postmortem human decomposition, but none describes detailed events between IUFD and delivery. We can, however, extrapolate basic theories about factors that accelerate human decomposition to those impacting fetal demise. Most notably, autolytic changes may occur faster under the increased temperature with maternal hyperthermia/sepsis associated with prolonged rupture of membranes. Similarly, if the fetal death is a result of fulminating bacterial infection (chorioamnionitis due to prolonged rupture of membranes), accelerated putrefaction (decomposition) can also be expected [23].

In community-based studies, verbal autopsy is used to estimate population-based rates of stillbirth by asking women when fetal movement stopped $[9,24]$. One study on the accuracy of verbal autopsy in 311 cases of stillbirth suggested that verbal autopsy and hospital-identified time of stillbirth were in concordance in $94 \%$ of prepartum deaths and $85 \%$ of intrapartum deaths, although the study used only fetal appearance of fresh versus macerated for some of the cases [9]. Ellis et al. [25] utilized verbal autopsy to compare maternal report of when fetal movement stopped with maternal description of the fetus as fresh or macerated. Of 201 fetuses moving when labor started, 24\% were described as macerated. Of the 117 fetuses not moving when labor started, 34\% were described as fresh.

The present study had several limitations. First, the quality of medical chart data in low-resource settings is variable and key data points 


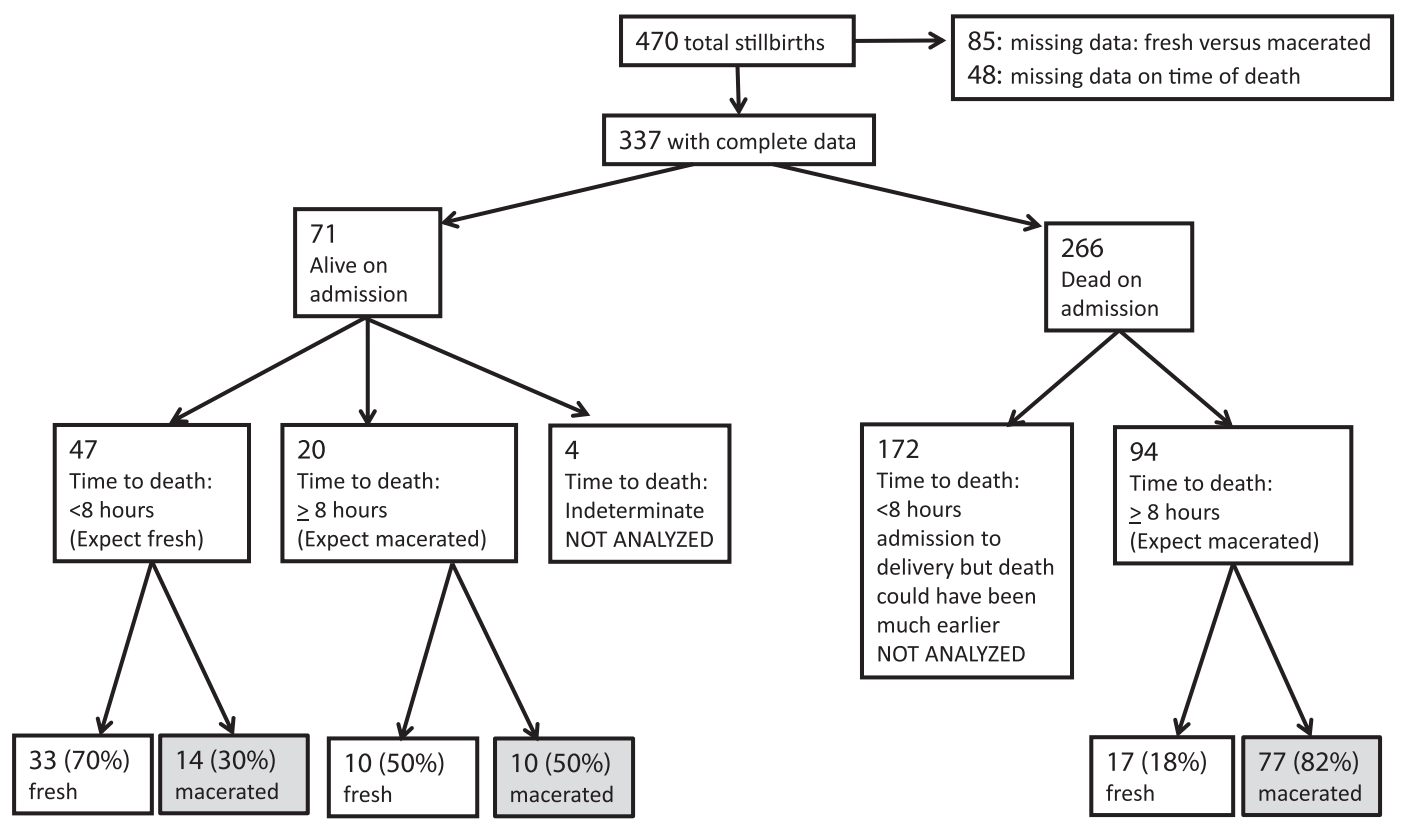

Fig. 2. Distribution of fresh and macerated stillbirths according to death-to-delivery interval.

were often not recorded, so the cases were excluded. This is a widespread barrier to data collection in low-income countries and reflects the reality of the working conditions and the challenges to conducting research in countries with the highest need for data. Second, the analysis was performed at a major hospital with 14000 deliveries each year. Like most hospitals in Sub-Saharan Africa with this volume of deliveries, there was no equipment for continuous monitoring of FHR, which would allow precise estimation of time of death. Third, there were a limited number of midwives and physicians at the study hospital and throughout Ghana so, even with intermittent monitoring, there can be long gaps between assessments of FHR. Fourth, no stillborn fetus in the present study had a postmortem autopsy, which would have allowed more precise identifications of time of death and fetal appearance.

Strengths of the study included the capture of all stillbirths over a 1-year period at a large urban hospital, as well as collection of systematic data on maternal demographics and risk factors, pregnancy care, delivery complications, and hospital variables. Few hospital-based studies collect such detailed information, despite it enabling richer understanding of these deaths.

The present large and comprehensive study to assess fetal appearance as a predictor of death-to-delivery interval indicates that such practice may be problematic. Although intrapartum fetal demise has been seen as a prime target for stillbirth prevention, we would caution against assuming that fetal appearance accurately indicates intrapartum deaths. Health facilities in low-income countries have enormous resource challenges to providing clinical care, and data collection is often a low priority. However, as the present study shows, medical records that identify accurate timing of positive FHR and diagnosis of fetal demise could provide critical data to focus efforts toward measuring and improving stillbirth outcomes worldwide.

\section{Acknowledgments}

Funding for travel was provided by grants from the University of Michigan Global REACH office and the University of Michigan Institute for Research on Women and Gender. K.J.G. received salary support from the National Institutes of Health as part of a K-23 training grant. No funder had a role in analysis of results or preparation/review of the manuscript.

\section{Conflict of interest}

The authors have no conflicts of interest.

\section{References}

[1] Lawn JE, Gravett MG, Nunes TM, Rubens CE, Stanton C, GAPPS Review Group. Global report on preterm birth and stillbirth (1 of 7): definitions, description of the burden and opportunities to improve data. BMC Pregnancy Childbirth 2010;10(Suppl. 1):S1.

[2] Frøen JF, Gordijn SJ, Abdel-Aleem H, Bergsjø P, Betran A, Duke CW, et al. Making stillbirths count, making numbers talk - issues in data collection for stillbirths. BMC Pregnancy Childbirth 2009;9:58.

[3] Goldenberg RL, McClure EM, Bhutta ZA, Belizán JM, Reddy UM, Rubens CE, et al. Stillbirths: the vision for 2020. Lancet 2011;377(9779):1798-805.

[4] Lawn JE, Lee AC, Kinney M, Sibley L, Carlo WA, Paul VK, et al. Two million intrapartum-related stillbirths and neonatal deaths: where, why, and what can be done? Int J Gynecol Obstet 2009;107(Suppl. 1) S5-18, S19.

[5] Barros FC, Bhutta ZA, Batra M, Hansen TN, Victora CG, Rubens CE, et al. Global report on preterm birth and stillbirth (3 of 7): evidence for effectiveness of interventions. BMC Pregnancy Childbirth 2010;10(Suppl. 1):S3.

[6] Yakoob MY, Ali MA, Ali MU, Imdad A, Lawn JE, Van Den Broek N, et al. The effect of providing skilled birth attendance and emergency obstetric care in preventing stillbirths. BMC Public Health 2011;11(Suppl. 3):S7.

[7] McClure EM, Pasha O, Goudar SS, Chomba E, Garces A, Tshefu A, et al. Epidemiology of stillbirth in low-middle income countries: a Global Network Study. Acta Obstet Gynecol Scand 2011;90(12):1379-85.

[8] Aggarwal AK, Jain V, Kumar R. Validity of verbal autopsy for ascertaining the causes of stillbirth. Bull World Health Organ 2011;89(1):31-40.

[9] Edmond KM, Quigley MA, Zandoh C, Danso S, Hurt C, Owusu Agyei S, et al. Diagnostic accuracy of verbal autopsies in ascertaining the causes of stillbirths and neonatal deaths in rural Ghana. Paediatr Perinat Epidemiol 2008;22(5):417-29.

[10] Shanklin DR. Fetal Maceration. 2. An analysis of 53 human stillborn infants. Am J Obstet Gynecol 1964;88:224-9.

[11] Hossain N, Khan N, Khan NH. Obstetric causes of stillbirth at low socioeconomic settings. J Pak Med Assoc 2009;59(11):744-7.

[12] Okeudo C, Ezem B, Ojiyi E. Stillbirth rate in a teaching hospital in South-eastern Nigeria: a silent tragedy. Ann Med Health Sci Res 2012;2(2):176-9.

[13] Yatich NJ, Funkhouser E, Ehiri JE, Agbenyega T, Stiles JK, Rayner JC, et al. Malaria, intestinal helminths and other risk factors for stillbirth in Ghana. Infect Dis Obstet Gynecol 2010;2010:350763.

[14] World Health Organization. Maternal, newborn, child and adolescent health: Stillbirths. http://www.who.int/maternal_child_adolescent/epidemiology/stillbirth/en/. Accessed 2013.

[15] Langley FA. The perinatal postmortem examination. J Clin Pathol 1971;24(2): 159-69.

[16] Bove KE. Practice guidelines for autopsy pathology: the perinatal and pediatric autopsy. Autopsy Committee of the College of American Pathologists. Arch Pathol Lab Med 1997;121(4):368-76

[17] STROBE. STROBE Statement: checklist of items that should be included in reports of observational studies. http://www.strobe-statement.org/fileadmin/Strobe/uploads/ checklists/STROBE_checklist_v4_combined.pdf. Published 2007. Accessed 2013. 
[18] Lawn J, Shibuya K, Stein C. No cry at birth: global estimates of intrapartum stillbirths and intrapartum-related neonatal deaths. Bull World Health Organ 2005;83(6): 409-17.

[19] Wigglesworth JS. Monitoring perinatal mortality. A pathophysiological approach. Lancet 1980;2(8196):684-6.

[20] Genest DR, Singer DB. Estimating the time of death in stillborn fetuses: III. External fetal examination; a study of 86 stillborns. Obstet Gynecol 1992;80(4):593-600.

[21] Pinar H, Koch MA, Hawkins H, Heim-Hall J, Abramowsky CR, Thorsten VR, et al. The stillbirth collaborative research network postmortem examination protocol. Am J Perinatol 2012;29(3):187-202.
[22] Gilbert-Barness E. Potter's Pathology of the Fetus, Infant, and Child, vol. 1. Philadelphia, PA: Mosby Elsevier; 2007.

[23] Janaway RC. The decay of buried human remains and their associated materials. In: Hunter J, Roberts CA, Martin A, editors. Studies in Crime: An Introduction to Forensic Archaeology. East Sussex: Psychology Press; 1996. p. 58-85.

[24] Lawn JE, Blencowe H, Pattinson R, Cousens S, Kumar R, Ibiebele I, et al. Stillbirths: Where? When? Why? How to make the data count? Lancet 2011;377(9775):1448-63.

[25] Ellis M, Azad K, Banerjee B, Shaha SK, Prost A, Rego AR, et al. Intrapartum-related stillbirths and neonatal deaths in rural bangladesh: a prospective, communitybased cohort study. Pediatrics 2011;127(5):e1182-90. 